\title{
Diagnósticos de enfermagem do domínio Nutrição identificados em idosos da comunidade
}

\author{
Nursing diagnosis of the Nutrition domain identified among the elderly in the community
}

Diagnósticos de enfermería en el dominio Nutrición identificados en ancianos de la comunidad

Jorge Wilker Bezerra Clares ${ }^{1}$, Maria Célia de Freitas ${ }^{2}$

${ }^{1}$ Enfermeiro. Discente do Programa de Pós-Graduação Cuidados Clínicos em Enfermagem e Saúde, nível Mestrado, Universidade Estadual do Ceará

(UECE). Fortaleza, CE, Brasil. E-mail: jorgewilker clares@yahoo.com.br.

${ }^{2}$ Enfermeira, Doutora em Enfermagem. Professora Titular da UECE. Fortaleza, CE, Brasil. E-mail: celfrei@hotmail.com.

\section{RESUMO}

Estudo descritivo que objetivou identificar diagnósticos de enfermagem do domínio Nutrição, da Taxonomia II da NANDA-I, em idosos acompanhados no âmbito da atenção primária à saúde em Fortaleza, Ceará, Brasil. Participaram 52 sujeitos selecionados intencionalmente. Os dados foram coletados no domicílio do idoso em maio e junho de 2011 mediante aplicação de um histórico de enfermagem, segundo referencial teórico de Henderson. Para estabelecimento dos diagnósticos de enfermagem seguiu-se as etapas propostas por Risner. Foram identificados nove diagnósticos de enfermagem dentre os 14 previstos no domínio Nutrição, destacando-se: Risco de desequilíbrio eletrolítico (50\%), Risco de volume de líquidos deficiente (50\%) e Nutrição desequilibrada: menos do que as necessidades corporais (32,7\%). Esses diagnósticos poderão subsidiar ações de saúde/enfermagem com foco na nutrição do idoso com vistas ao atendimento das reais necessidades desse grupo, numa perspectiva integral e resolutiva.

Descritores: Idoso; Diagnóstico de Enfermagem; Nutrição do Idoso; Atenção Primária à Saúde; Enfermagem.

\section{ABSTRACT}

The objective of this descriptive study was to identify the nursing diagnosis under the Nutrition domain, according to NANDA International Taxonomy II, in elderly patients of primary health care units in Fortaleza, Ceará, Brazil. The intentional sample consisted of 52 subjects. Data collection was performed at the elderly's home, in May and June of 2011, by means of the application of a nursing history, following the theoretical framework proposed by Henderson. The nursing diagnoses were established following the stages proposed by Risner. Nine nursing diagnoses were identified among the 14 listed under the Nutrition domain. The main were: Risk for electrolyte imbalance (50\%), Risk for deficient fluid volume (50\%) and Imbalanced nutrition: less than body requirements (32.7\%). These diagnoses can support health/nursing interventions focused on elderly nutrition, aiming to meet the real needs of this group, from a comprehensive and resolving perspective.

Descriptors: Aged; Nursing Diagnosis; Elderly Nutrition; Primary Health Care; Nursing.

\section{RESUMEN}

Estudio descriptivo objetivando identificar diagnósticos de enfermería del dominio Nutrición, de la Taxonomía II de la NANDAI en ancianos en seguimiento en atención básica de salud en Fortaleza, Ceará, Brasil. Participaron 52 sujetos, seleccionados intencionalmente. Datos recolectados en los domicilios de los ancianos, entre mayo y junio de 2011, mediante aplicación de un histórico de enfermería, según referencial teórico de Henderson. Para establecer los diagnósticos de enfermería, se siguieron las etapas propuestas por Risner. Fueron identificados nueve diagnósticos de enfermería de los catorce previstos en el dominio Nutrición, destacándose: Riesgo de desequilibrio electrolítico (50\%), Déficit de volumen de líquidos (50\%) y Desequilibrio nutricional: ingesta inferior a las necesidades (32,7\%). Dichos diagnósticos podrán resultar de ayuda en acciones de salud/enfermería con foco en la nutrición del anciano, en procura de la atención de las necesidades reales de ese grupo, en una perspectiva integral y resolutiva.

Descriptores: Anciano; Diagnóstico de Enfermería; Nutricion del Idoso; Atención Primaria de Salud; Enfermería. 


\section{INTRODUÇÃO}

A nutrição é um elemento importante da saúde da população idosa e afeta o processo de envelhecimento. As progressivas alterações decorrentes do processo de envelhecimento provocam diversas modificações funcionais e estruturais de órgãos e tecidos que refletem em todo organismo do idoso e podem afetar o seu estado nutricional, tais como: a redução da massa corporal magra, alterações nos níveis de citocinas e hormonal, retardo no esvaziamento gástrico, olfato e paladar diminuídos, dentre outros ${ }^{(1)}$. Além disso, doenças crônicas não transmissíveis (DCNT), estado emocional, medicamentos e isolamento social podem acarretar desequilíbrio nutricional nos idosos(2), expondo-os a um risco aumentado para infecção, hospitalizações, mortalidade e prejuízos na qualidade de vida(3-4).

As últimas estimativas sugerem que 1,3 milhões de pessoas acima de 65 anos sofrem de desnutrição, e a grande maioria (93\%) vive na comunidade ${ }^{(5)}$. Da mesma forma, a obesidade tem sido considerada um sério problema de saúde pública, atingindo índices cada vez mais elevados entre os idosos no Brasil e no mundo(6). Portanto, o estado nutricional do idoso pode ser considerado um importante indicador de seu estado geral de saúde.

Esses dados chamam atenção para a necessidade de que os profissionais de saúde, em especial os enfermeiros, assumam o compromisso de oferecer à população idosa uma atenção integral, incluindo a avaliação nutricional na avaliação multidimensional de saúde dos idosos, a fim de prevenir e tratar o estado nutricional inadequado e garantir um atendimento de alta qualidade a essa população.

O enfermeiro enquanto membro da equipe multidisciplinar e líder da equipe de enfermagem assume papel de destaque no cuidado ao idoso, devendo atuar com foco na promoção, educação, manutenção e recuperação da saúde do ser que envelhece ou dos que já se encontram no processo de envelhecimento(7). Esse profissional deve se fundamentar em conhecimento científico e na autonomia profissional; e para alcançar esta meta suas ações devem ser sistematizadas por meio do processo de enfermagem, proporcionando a melhoria da qualidade dos cuidados de saúde/enfermagem e estimulando a construção de conhecimentos teóricos e científicos com base nas melhores práticas de enfermagem ${ }^{(8)}$.

Para a eficácia e efetividade da prática de enfermagem torna-se essencial trabalhar com um sistema padronizado de linguagem para os fenômenos de enfermagem. Nesse sentido, a North American Nursing Diagnosis Association Internacional (NANDA-I) tem desenvolvido um sistema conceitual para classificar os diagnósticos de enfermagem em uma taxonomia. A Taxonomia II da NANDA-I é reconhecida como uma fonte consolidada de terminologia de diagnóstico de enfermagem e compreende três níveis: domínios, classes e diagnósticos de enfermagem, totalizando 13 domínios, 47 classes e 201 diagnósticos. O domínio Nutrição compreende classes e diagnósticos de enfermagem relacionados às atividades de ingerir, assimilar e usar nutrientes com fins de manter e reparar tecidos e produzir energia ${ }^{(9)}$.

Essa taxonomia possibilita ao enfermeiro interpretar, analisar e julgar os problemas de saúde reais ou de risco, baseados na manifestação das respostas humanas dos pacientes. Os diagnósticos tornam-se importantes para a prática do enfermeiro, pois padronizam uma linguagem para a profissão e conjuntamente com o processo de enfermagem refletem a variedade, a complexidade e a cientificidade do cuidar, demonstrando com clareza um compromisso para unir teoria, educação e prática clínica de enfermagem ${ }^{(9)}$. Portanto, a utilização de uma linguagem diagnóstica possibilita integrar e guiar as ações de enfermagem, qualificar e humanizar o cuidado prestado ao idoso na comunidade.

Diante do exposto, considera-se fundamental a realização de estudos sobre a investigação do estado nutricional do idoso e a necessidade de incluí-la na avaliação multidimensional de saúde, reforçando a participação do enfermeiro nesse processo, por meio da identificação de problemas/demandas que servirão de base para a elaboração dos diagnósticos de enfermagem. Acredita-se que esses dados poderão subsidiar o planejamento e intervenções eficazes, contribuindo para a prestação de uma assistência humanizada e resolutiva ao idoso e favorecendo a atuação do enfermeiro junto à equipe interdisciplinar, no âmbito da atenção primária.

Posto isso, o objetivo desta investigação foi identificar os diagnósticos de enfermagem do domínio Nutrição, segundo a Taxonomia II da NANDA-I, em idosos 
acompanhados no âmbito da atenção primária à saúde, em Fortaleza-CE.

\section{MÉTODOS}

Estudo descritivo, de corte transversal, realizado em uma Unidade de Saúde da Família (USF) de Fortaleza, Ceará, Brasil. Trata-se de uma unidade modelo, referência no atendimento e acompanhamento da população no âmbito da atenção primária à saúde; possui cinco equipes de saúde da família, distribuídas nas oito microáreas que compõem o território adscrito à USF. Destas microáreas, duas estão localizadas na área que integra o campus de uma universidade pública, sendo local de prática para atividades de ensino, pesquisa e extensão dos cursos da área da saúde da referida instituição.

A população do estudo foi composta pelos idosos residentes no território de abrangência dessas duas microáreas, perfazendo um total de 61 idosos, segundo dados do último censo realizado pelos agentes comunitários de saúde (ACS), durante a campanha de vacinação contra a gripe, que ocorreu no período de 25 de abril a 13 de maio de 2011. Foram excluídos os idosos não encontrados no domicílio após três tentativas de visita. Dessa forma, a amostra final foi composta por 52 idosos.

O projeto foi encaminhado e aprovado pelo Comitê de Ética em Pesquisa do Hospital de Messejana Dr. Carlos Alberto Studart Gomes, sob o número de protocolo $797 / 10$. Рага a entrada em campo pelos pesquisadores houve ainda aquiescência da Secretaria Municipal de Saúde de Fortaleza e da gestão local.

Para a coleta de informações, elaborou-se um instrumento cuja organização e estruturação foram fundamentadas na teoria de enfermagem de Virgínia Henderson $^{(10)}$, subsidiando o levantamento de informações (histórico de enfermagem). O referido instrumento constou dos seguintes itens: dados socioeconômicos (sexo, idade, estado civil, grau de instrução, ocupação e renda) e informações acerca das 14 necessidades humanas fundamentais, segundo a referida teórica, a saber: respiração, nutrição/hidratação, eliminação, sono/repouso, movimento, vestuário, higiene/proteção, termorregulação, segurança, trabalho/realização, religião/espiritualidade, comunicação, lazer, aprendizagem ${ }^{(10)}$. No presente estudo, foram analisadas as questões pertinentes à necessidade de nutrição/hidratação, segundo Henderson, cujo enfoque foi para a investigação da presença de problemas gástricos, dificuldade de engolir, quantidade de líquidos ingeridos/dia, número de refeições diárias, dificuldades no preparo das refeições e a necessidade de ajuda para comer e/ou beber.

Inicialmente, foram aplicados testes pilotos com quatro idosos (aproximadamente $10 \%$ da amostra) com características semelhantes ao grupo estudado, e feito reformulações no instrumento para melhor compreensão dos participantes. Ressalta-se que esses sujeitos não foram incluídos na amostra do estudo.

Os dados foram coletados nos meses de maio e junho de 2011, no domicílio dos idosos. Todos os participantes foram esclarecidos quanto aos objetivos e procedimentos envolvidos no estudo, ao direito de recusar-se ou desistir da participação na pesquisa e à preservação do anonimato, e aqueles que concordaram manifestaram o aceite por escrito, por meio do preenchimento do termo de consentimento livre e esclarecido.

A partir das informações obtidas mediante aplicação dos formulários, procedeu-se ao processo de julgamento clínico, o qual seguiu as etapas propostas por Risner ${ }^{(11)}$, levando em consideração as maiores dificuldades e limitações dos idosos em satisfazer a necessidade de nutrição/hidratação. A identificação das afirmativas diagnósticas do domínio Nutrição, segundo a Taxonomia II da NANDA-I ${ }^{(9)}$, foi feita por dois pesquisadores (discente e docente) e não houve divergência de opiniões.

Os dados foram armazenados em um banco eletrônico criado no Microsoft ${ }^{\circledR}$ Excel 2007, utilizando a técnica de dupla verificação e digitação para reduzir possíveis erros na transcrição das informações. Para análise dos resultados foram utilizados procedimentos de estatística descritiva, com distribuição das frequências absolutas e relativas. Os dados foram descritos quantitativamente e discutidos à luz da literatura pertinente.

\section{RESULTADOS}

Participaram do estudo 52 idosos: 36 (69,2\%) eram do sexo feminino, $20(38,5 \%)$ com idade entre 60 e 69 anos, 25 (48,1\%) casados/união estável, 36 (69,2\%) aposentados, $46 \quad(88,5 \%)$ apresentaram baixa escolaridade e $50(96,2 \%)$ com renda inferior a três salários mínimos, considerando o vigente à época: $\mathrm{R} \$ 545,00$. 
Quanto às morbidades relatadas, os idosos referiram hipertensão arterial (48,1\%), osteoporose (34,2\%), alcoolismo $(21,2 \%)$, diabetes $(19,2 \%)$, gastrite $(15,4 \%)$ e outros, com ocorrência menor, como reumatismo, depressão, insuficiência cardíaca, insuficiência renal crônica, doença de Parkinson e doença de Alzheimer. Destaca-se que 12 (23,1\%) dos idosos faziam uso de diuréticos para o controle dos níveis pressóricos.

Em relação aos fatores que interferem na nutrição/hidratação do idoso, a grande maioria (92,3\%) afirmou não necessitar de ajuda para comer e/ou beber, todavia, esses dados divergem de alguns achados que revelaram predisposição dessa população à dependência para satisfazê-la, sejam os quais: problemas gástricos (36,5\%), disfagia $(19,2 \%)$, dentição incompleta $(96,2 \%)$, ingestão de líquidos $(32,7 \%)$ e número de refeições $(46,1 \%)$ menores que as quantidades recomendadas e dificuldade no preparo das refeições $(25,0 \%)$.

No domínio Nutrição, foram identificados nove diagnósticos dentre os 14 previstos na Taxonomia II da NANDA-I, os quais são apresentados na Tabela 1. Destes, quatro são do tipo real e cinco do tipo risco.

Tabela 1: Distribuição dos diagnósticos de enfermagem do domínio Nutrição, identificados nos idosos cadastrados numa USF do nordeste brasileiro. Fortaleza, CE, Brasil, 2011.

\begin{tabular}{lcc}
\hline \multicolumn{1}{c}{ Diagnósticos de enfermagem } & N & \% \\
\hline Risco de desequilíbrio eletrolítico & 26 & 50,0 \\
Risco de volume de líquidos deficiente & 26 & 50,0 \\
Nutrição desequilibrada: menos do que as necessidades corporais & 17 & 32,7 \\
Risco de disfunção hepática & 12 & 23,1 \\
Risco de glicemia instável & 11 & 21,7 \\
Deglutição prejudicada & 10 \\
Risco de nutrição desequilibrada: mais do que as necessidades corporais & 19,2 \\
Nutrição desequilibrada: mais do que as necessidades corporais & 07 \\
Volume de líquidos excessivo & 13,5 \\
\hline
\end{tabular}

\section{DISCUSSÃO}

O enfermeiro atuante na atenção primária deve considerar que um estado nutricional equilibrado favorece a saúde física e emocional, prevenindo ou postergando o surgimento das afecções mais frequentes entre os idosos ${ }^{(12)}$. De tal modo, esse profissional deverá tentar, por meio de ações sistematizadas, corrigir ou prevenir os problemas resultantes do excesso ou deficiências relacionadas à nutrição e à hidratação, levando em conta o nível socioeconômico do idoso, sua capacidade funcional, seu poder aquisitivo para adquirir os alimentos e sua carga de doenças. Para alcançar estas metas, necessita de conhecimento ampliado para realizar um julgamento clínico eficiente na formulação de diversos diagnósticos de enfermagem, que possam subsidiar a escolha das intervenções mais adequadas.

Dentre os diagnósticos de enfermagem elaborados, os de risco revelaram maior evidência na população em estudo, sendo apresentados em mais da metade dos participantes. Os diagnósticos de risco descrevem respostas humanas a condições de saúde/processos vitais que podem desenvolver-se em indivíduo, família ou comunidade vulnerável. Estão apoiados em fatores de risco que contribuem para o aumento da vulnerabilidade, principalmente quando a clientela é de idosos que têm alterações decorrentes do processo de envelhecimento. Os diagnósticos reais ou atuais, por sua vez, descrevem respostas humanas a condições de saúde/processos vitais que existem em um idoso, na família cuidadora, bem como na comunidade onde residem. É sustentado pelas características definidoras (manifestações, sinais e sintomas), que se agrupam em padrões de indícios ou inferências relacionados ${ }^{(9)}$.

Dentre os diagnósticos de enfermagem que mais se destacaram, o risco de volume de líquidos deficiente e o risco de desequilíbrio eletrolítico apresentaram as maiores frequências, sendo encontrados em $50 \%$ da amostra, cada.

Considera-se que devido ao uso de diuréticos e a ingesta líquida menor que a quantidade mínima recomendada, inevitavelmente ocorre a exposição aumentada do indivíduo a desordens do equilíbrio hidroeletrolítico. Além disso, fatores como extremo de idade, desvios que afetam a ingestão e/ou absorção de líquidos e as perdas excessivas de líquidos por vias normais (p.ex., diarreia), também foram observados, caracterizando o risco de volume de líquidos deficiente, 
definido como "risco de desidratação vascular, celular ou intracelular" (9).

Devido ao processo de envelhecimento ocorrem alterações na regulação da sede, reduzindo o estímulo da sede e, consequentemente, limitando a ingestão de líquidos pelo idoso. Portanto, chama a atenção o fato de $32,7 \%$ dos participantes consumirem menos líquidos que a quantidade mínima recomendada. Entre os idosos, é indicado um aporte diário de líquidos de, no mínimo, dois litros, uma vez que nessa faixa etária há um risco mais acentuado para desidratação devido à redução da taxa de filtração glomerular ${ }^{(12)}$. Acrescenta-se que as alterações que comprometem o teor hídrico no organismo, além de desidratação, podem levar a prejuízos na concentração de eletrólitos séricos, predispondo o indivíduo ao risco de desequilíbrio eletrolítico, definido como o "risco de mudança nos níveis eletrolíticos séricos, capaz de comprometer a saúde"(9).

Esses diagnósticos exigem uma atenção especial por parte dos enfermeiros das equipes de saúde da família, que devem identificar as causas da ingesta hídrica insuficiente e/ou da perda líquida excessiva, e desenvolver um plano de cuidados que resulte na redução de comportamentos de risco e adoção de comportamentos promotores de saúde pelo idoso. No âmbito da atenção primária, são sugeridas as seguintes ações de enfermagem: realizar exame físico completo, inspecionar a presença de edema, avaliar o aspecto de pele e mucosas, incentivar uma ingesta hídrica adequada, e observar sinais de alterações clínicas, como fraqueza, diarreia, vômitos, anúria/oligúria, desidratação e alterações de comportamento(13).

O "estado no qual um indivíduo está experimentando uma ingestão insuficiente de nutrientes para satisfazer as necessidades metabólicas"(9) caracteriza o diagnóstico nutrição desequilibrada: menos do que as necessidades corporais, identificado em $32,7 \%$ dos sujeitos pesquisados. Resultados semelhantes foram encontrados em estudo realizado com idosas que vivem em comunidades de baixa renda de Jequié, Bahia, cuja prevalência de risco para desnutrição ou desnutridas foi de $33,8 \%{ }^{(14)}$. Pesquisa realizada com 238 idosos residentes nas áreas rurais de Olésnica, Polônia, evidenciou que todos os participantes apresentaram deficiências nutricionais significativas ${ }^{(15)}$.
Os hábitos alimentares de uma pessoa possuem forte associação ao seu padrão socioeconômico, rendimento e estilo de vida. Nesse contexto, autores reforçam que os orçamentos insuficientes e o custo elevado dos alimentos obrigam os idosos a economizar e a reduzir a quantidade e a qualidade dos insumos alimentares(12).

Além do fator econômico, a restrição da dieta do idoso pode ser ocasionada em razão dos processos de adoecimento crônico, tais como hipertensão arterial, diabetes, doenças cardíacas, problemas gastrintestinais e osteoarticulares, que requerem o seguimento de regimes alimentares especiais e consumo de medicamentos que, muitas vezes, fogem aos parcos recursos financeiros dos idosos.

No presente estudo, os idosos aposentados apresentaram um melhor padrão alimentar, com maior número de refeições diárias, do que aqueles que ainda não adquiriram esse benefício. Considerando que a população investigada reside em uma área periférica da capital cearense, com baixos indicadores socioeconômicos, pode-se inferir que a aposentadoria representou uma melhoria das condições de vida daqueles idosos, garantindo-lhes um rendimento mínimo para o atendimento de suas necessidades fundamentais, dentre eles um melhor padrão alimentar.

A dificuldade no preparo das refeições, manifestada por $25 \%$ dos idosos, implica em importantes consequências na manutenção da autonomia e independência desses sujeitos, gerando impacto negativo em sua qualidade de vida. Portanto, os idosos com prejuízos na autonomia tornam-se menos capazes de tomar conta de si e, não raro, acabam por negligenciar a alimentação, podendo ocasionar graves problemas de desnutrição(12).

É necessário que o enfermeiro, durante as consultas de enfermagem realizadas na USF ou durante as visitas domiciliárias, investigue aspectos relacionados à alimentação da pessoa idosa. Nesse sentido, o profissional deve estar atento para: perda da capacidade/autonomia para comprar e/ou preparar os alimentos e para alimentar-se; perda de apetite e redução da sensação de sede e da percepção da temperatura dos alimentos; perda parcial ou total da visão que dificulte a seleção, preparo e consumo dos alimentos; perda ou redução da capacidade olfativa, interferindo no seu apetite; dietas restritivas; alterações de peso recentes; 
dificuldade de mastigação por lesão oral, uso de prótese dentária ou problemas digestivos; dificuldade de deglutição, dentre outros aspectos relevantes. Essas informações poderão auxiliar o enfermeiro no estabelecimento de estratégias para melhorar o padrão alimentar e nutricional dos idosos, levando em consideração o contexto sociocultural onde essa população está inserida(13).

O diagnóstico de enfermagem risco de função hepática prejudicada foi encontrado em $23,1 \%$ da amostra e teve o uso abusivo e crônico do álcool como principal fator de risco. O consumo do álcool fornece calorias vazias de proteínas, vitaminas e sais minerais, reduz a sensação de fome, piorando a desnutrição, não só por diminuir a ingestão de alimentos, como também por alterar a digestão e absorção do ingerido e a ativação das vitaminas a nível hepático(16). O enfrentamento dessa situação requer treinamento dos enfermeiros para identificar os problemas e realizar intervenções apropriadas a fim de potencializar a recuperação do usuário alcoolista. Esse profissional deve utilizar estratégias de aproximação com o idoso, incentivando sua participação em grupos de apoio para orientação, acolhimento e outras estratégias terapêuticas, com vistas às mudanças de comportamento e à adoção de um estilo de vida mais saudável.

O risco de glicemia instável, definido como uma situação de "risco de variação dos níveis de glicose no sangue em relação aos parâmetros normais"(9), foi encontrado em $21,7 \%$ da amostra. Para esse diagnóstico, os principais fatores de risco encontrados foram: aumento de peso, ingestão alimentar inadequada, conhecimento deficiente do diabetes e/ou falta de aceitação do diagnóstico, não adesão ao seu controle/regime terapêutico e baixo nível de atividade física.

Nos diabéticos, que correspondem a 19,2\% dos entrevistados, essa situação é mais recorrente, uma vez que quando a doença não é controlada adequadamente pode resultar em episódios de hipo/hiperglicemia a depender de suas características e hábitos de saúde. Рara o controle eficaz dos níveis glicêmicos, é preciso uma atuação conjunta entre idoso e profissional de saúde, por meio da qual o enfermeiro deve fornecer orientações, esclarecer dúvidas e traçar um plano de ação que vise um estilo de vida mais saudável, contemplando, entre outros aspectos, o uso correto da medicação, a reeducação alimentar e a prática de atividade física regular ${ }^{(17)}$.

A situação de "funcionamento anormal do mecanismo de deglutição associado a déficit na estrutura ou função oral, faríngea ou esofágica"(9) define o diagnóstico deglutição prejudicada, que esteve presente em 19,2\% dos idosos. Teve a dentição prejudicada como principal fator relacionado. A dentição incompleta pode prejudicar na deglutição dos alimentos, em virtude da associação entre salivação insuficiente, dificuldade de mastigação e redução da motilidade digestiva, podendo resultar em desnutrição, perdas importantes de peso ou ainda risco de broncoaspiração(12). O elevado número de dentes extraídos encontrado neste levantamento, à semelhança de outros estudos, evidencia a precariedade da situação de saúde bucal dos idosos brasileiros e a inexistência de tratamento restaurador ao alcance da maioria da população(18).

No Brasil, a assistência pública odontológica a idosos precisa ser incrementada, por meio da elaboração de programas que incluam ações educativas voltadas para o autocuidado, além de ações preventivas e reabilitadoras. No contexto da atenção primária, a equipe de enfermagem pode atuar juntamente com as equipe de saúde bucal, por meio de um trabalho interdisciplinar, a fim de identificar os principais problemas relacionados à saúde bucal dos idosos de sua comunidade adscrita e desenvolver estratégias para superá-los.

Os diagnósticos de enfermagem risco de nutrição desequilibrada: mais do que as necessidades corporais e nutrição desequilibrada: mais do que as necessidades corporais estiveram presentes em 13,5\% e 11,6\% da amostra, respectivamente. Esses diagnósticos referem-se a uma situação de risco ou instalada na qual o indivíduo ingere uma quantidade de nutrientes superior a suas necessidades metabólicas ${ }^{(9)}$. Esses sujeitos se encontravam em sobrepeso/obesidade ou em risco para essas condições, e não realizavam atividade física regular.

O excesso de peso tem sido considerado um sério problema de saúde pública, atingindo idosos de todo o mundo. Nos Estados Unidos, entre 2007-2010, mais de um terço dos idosos com 65 anos ou mais de idade eram obesos, o que corresponde a cerca de 13 milhões de pessoas $^{(19)}$. No contexto brasileiro, estudo realizado com idosos atendidos pela ESF de Passo Fundo, Rio Grande do 
Sul, evidenciou prevalência de $49,6 \%$ para sobrepeso/obesidade ${ }^{(6)}$.

Diante dessa realidade, os enfermeiros devem atuar junto à população idosa incentivando a adoção de mudanças para promover um estilo de vida mais saudável, incluindo auxiliá-los a superar os obstáculos relacionados com modificações na dieta, prática de exercícios físicos regulares, uso de suportes comunidade e prevenção de agravos à saúde ${ }^{(20)}$.

O diagnóstico volume de líquidos excessivo, definido como a "retenção aumentada de líquidos isotônicos"(9), se destacou em 9,6\% dos idosos e esteve relacionado principalmente à ingesta excessiva de sódio, mecanismos reguladores comprometidos (disfunção renal), insuficiência cardíaca congestiva e alterações vasculares, produzindo edema. Relativamente a esse diagnóstico, compete aos enfermeiros da atenção primária a contribuição para minimizar o edema local ou generalizado, criando meios para a compreensão do idoso acerca de seu estado de saúde e do risco de complicações relacionadas a esta situação clínica, implementando uma avaliação de enfermagem completa e estimulando a adoção de comportamentos desejáveis para uma boa saúde.

\section{CONCLUSÃO}

Com o presente estudo foi possível identificar os diagnósticos de enfermagem do domínio Nutrição em idosos de uma comunidade atendida no âmbito da atenção primária de saúde. O percentual acentuado de diagnósticos de risco indica que os problemas identificados são, em grande parte, situações potenciais que podem se instalar caso não haja uma intervenção adequada por parte da equipe de saúde.

Esta pesquisa apresentou como principais limitações o pequeno tamanho amostral e o delineamento seccional, o que impediu que as demais etapas do processo de enfermagem fossem executadas. Contudo, traz contribuições importantes para o cuidado ao idoso na atenção primária no que se refere à avaliação do estado nutricional, que, muitas vezes, não é contemplada durante as consultas de saúde/enfermagem. Acredita-se que esses diagnósticos poderão subsidiar o planejamento de intervenções com vistas ao atendimento das reais necessidades desse grupo e fundamentadas em conhecimento científico e na linguagem própria da Enfermagem.

Diante do exposto, sugere-se que a investigação dos aspectos relacionados à nutrição seja incluída na avaliação multidimensional de saúde do idoso realizada pelos enfermeiros e demais profissionais de saúde da família. Além disso, incentiva-se a realização de mais estudos sobre a temática a fim de contribuir para a produção de conhecimentos que possam refletir em melhores práticas de saúde no âmbito da atenção primária, com vistas a uma assistência integral e resolutiva à pessoa idosa.

[acesso em: 20 dez 2013];27(4):481-8. Disponível em: http://dx.doi.org/10.1016/j.clnu.2008.04.011.

5. Elia M, Smith RM. Improving nutritional care and treatment Perspectives and Recommendations from Population Groups, Patients and Carers [Internet]. Worcs: BAPEN; 2009 [acesso em: 20 dez 2013]. Disponível em:

http://www.bapen.org.uk/pdfs/improv nut care report.pdf. 6. Kümpel DA, Sodré AC, Pomatti DM, Scortegagna HM, Filippi J, Portella MR, et al. Obesidade em idosos acompanhados pela estratégia de saúde da família. Texto contexto - enferm [Internet]. 2011 [acesso em: $20 \mathrm{dez} 2013$ ];20(3):471-7. Disponível em: http://dx.doi.org/10.1590/S010407072011000300007.

7. Martins JJ, Barra DCC, Santos TM, Hinkel V, Nascimento ERP, Albuquerque GL, Erdmann AL. Educação em saúde como suporte para a qualidade de vida de grupos da terceira idade. Rev. Eletr. Enf. [Internet]. 2007 [acesso em: 20 dez 2013];9(2):443-56. Disponível em:

http://www.fen.ufg.br/revista/v9/n2/v9n2a12.htm. 8. Figueiredo MLF, Luz MHBA, Brito CMS, Sousa SNS, Silva DRS. Diagnósticos de enfermagem do idoso acamado no domicílio. Rev Bras Enferm [Internet]. 2008 [acesso em: $20 \mathrm{dez}$ 
2013];61(4):464-9. Disponível em:

http://dx.doi.org/10.1590/S0034-71672008000400011.

9. NANDA International. Diagnósticos de Enfermagem da Nanda

- Definições e Classificação 2009-2011. Porto Alegre: Artmed;

2010.

10. Organizacion Panamericana de la Salud. Principios basicos de los cuidados de enfermería [Internet]. Washington:

Organización Panamericana de la Salud; 1961 [acesso em: 20

dez 2013]. Disponível em:

http://hist.library.paho.org/English/SPUB/41741.pdf.

11. Risner PB. Diagnosis: analysis and synthesis of data. In: Cristensen PJ, Kenney JW, organizadoras. Nursing process application of conceptual models. St. Louis: Mosby; 1995. p. 12449.

12. Berger L, Mailloux-Poirier D. Pessoas idosas: uma abordagem global. Lisboa: Lusodidacta; 1995.

13. Ministério da Saúde. Envelhecimento e saúde da pessoa idosa [Internet]. Brasília: Ministério da Saúde; 2007 [acesso em: 20 dez 2013]. Disponível em:

http://bvsms.saude.gov.br/bvs/publicacoes/abcad19.pdf.

14. Virtuoso-Júnior JS, Tribess S, Romo-Perez V, Oliveira-Guerra

R. Factors associated to risk of malnutrition amongst elderly women in low-income communities. Colomb Med [Internet]. 2012 [acesso em: 20 dez 2013];43(1):54-62. Disponível em: http://colombiamedica.univalle.edu.co/index.php/comedica/arti cle/view/1059.

15. Wyka J, Biernat J, Mikołajczak J, Piotrowska E. Assessment of dietary intake and nutritional status (MNA) in Polish freeliving elderly people from rural environments. Arch Gerontol Geriatr [Internet]. 2012 [acesso em: $20 \mathrm{dez}$ 2013];54(1):44-9. Disponivel em: http://dx.doi.org/10.1016/j.archger.2011.02.001. 16. Acauan L, Donato M, Domingos AM. Alcoolismo: um novo desafio para o enfermeiro. Esc. Anna Nery [Internet]. 2008 [acesso em: 20 dez 2013];12(3):566-70. Disponível em: http://dx.doi.org/10.1590/S1414-81452008000300026. 17. Shafiee G, Mohajeri-Tehrani M, Pajouhi M, Larijani B. The importance of hypoglycemia in diabetic patients. J Diabetes Metab Disord [Internet]. 2012 [acesso em: $20 \mathrm{dez}$ 2013];11(1):17. Disponível em: http://dx.doi.org/10.1186/22516581-11-17.

18. Martins AMEBL, Barreto SM, Silveira MF, Santa-Rosa TTA, Pereira RD. Self-perceived oral health among Brazilian elderly individuals. Rev Saude Publica [Internet]. 2010 [acesso em: 20 dez 2013];44(5):912-22. Disponível em:

http://dx.doi.org/10.1590/S0034-89102010005000028.

19. Fakhouri THI, Ogden CL, Carroll MD, Kit BK, Flegal KM.

Prevalence of Obesity Among Older Adults in the United States, 2007-2010. NCHS Data Brief [Internet]. 2012 [acesso em: $20 \mathrm{dez}$ 2013];(106). Disponível em:

http://www.cdc.gov/nchs/data/databriefs/db106.htm.

20. Newman A. Obesity in Older Adults. OJIN: The Online Journal of Issues in Nursing [Internet]. 2009 [acesso em: $20 \mathrm{dez}$ de 2013];14(1). Disponível em:

http://www.nursingworld.org/MainMenuCategories/ANAMarke tplace/ANAPeriodicals/OJIN/TableofContents/Vol142009/No1J an09/Obesity-in-Older-Adults.html.

Artigo recebido em 28/08/2012.

Aprovado para publicação em 18/10/2013.

Artigo publicado em 31/12/2013. 